\title{
Reductions in dead space ventilation with nasal high flow depend on physiological dead space volume: metabolic hood measurements during sleep in patients with COPD and controls
}

\author{
Paolo Biselli ${ }^{1,2}$, Kathrin Fricke ${ }^{1}$, Ludger Grote ${ }^{3}$, Andrew T. Braun ${ }^{1}$, \\ Jason Kirkness ${ }^{1}$, Philip Smith ${ }^{1}$, Alan Schwartz ${ }^{1}$ and Hartmut Schneider ${ }^{1}$ \\ Affiliations: ${ }^{1}$ Sleep Disorders Center, School of Medicine, Johns Hopkins University, Baltimore, MD, USA. \\ ${ }^{2}$ University Hospital, University of São Paulo, São Paulo, Brazil. ${ }^{3}$ Sleep Disorders Center, Sahlgrenska \\ Hospital, University of Gothenburg, Gothenburg, Sweden.
}

Correspondence: Paolo Biselli, School of Medicine, University of São Paulo - Medicine, Avenida Professor Lineu Prestes, 2565 São Paulo, SP 05508-000, Brazil. E-mail: p biselliahotmail.com

@ERSpublications

Nasal high flow decreases dead space ventilation in both controls and patients with COPD during sleep http://ow.ly/uJHd30jAzwA

Cite this article as: Biselli P, Fricke K, Grote L, et al. Reductions in dead space ventilation with nasal high flow depend on physiological dead space volume: metabolic hood measurements during sleep in patients with COPD and controls. Eur Respir J 2018; 51: 1702251 [https://doi.org/10.1183/13993003.02251-2017].

ABSTRACT Nasal high flow (NHF) reduces minute ventilation and ventilatory loads during sleep but the mechanisms are not clear. We hypothesised NHF reduces ventilation in proportion to physiological but not anatomical dead space.

11 subjects (five controls and six chronic obstructive pulmonary disease (COPD) patients) underwent polysomnography with transcutaneous carbon dioxide $\left(\mathrm{CO}_{2}\right)$ monitoring under a metabolic hood. During stable non-rapid eye movement stage 2 sleep, subjects received NHF $\left(20 \mathrm{~L} \cdot \mathrm{min}^{-1}\right)$ intermittently for periods of 5-10 min. We measured $\mathrm{CO}_{2}$ production and calculated dead space ventilation.

Controls and COPD patients responded similarly to NHF. NHF reduced minute ventilation (from $5.6 \pm 0.4$ to $4.8 \pm 0.4 \mathrm{~L} \cdot \mathrm{min}^{-1} ; \mathrm{p}<0.05$ ) and tidal volume (from $0.34 \pm 0.03$ to $0.3 \pm 0.03 \mathrm{~L} ; \mathrm{p}<0.05$ ) without a change in energy expenditure, transcutaneous $\mathrm{CO}_{2}$ or alveolar ventilation. There was a significant decrease in dead space ventilation (from $2.5 \pm 0.4$ to $1.6 \pm 0.4 \mathrm{~L} \cdot \mathrm{min}^{-1}$; $\mathrm{p}<0.05$ ), but not in respiratory rate. The reduction in dead space ventilation correlated with baseline physiological dead space fraction $\left(\mathrm{r}^{2}=0.36\right.$; $\mathrm{p}<0.05)$, but not with respiratory rate or anatomical dead space volume.

During sleep, NHF decreases minute ventilation due to an overall reduction in dead space ventilation in proportion to the extent of baseline physiological dead space fraction. 


\section{Introduction}

Nasal high flow (NHF) is a method of ventilatory support increasingly used in several clinical settings. During NHF, warm and humidified air is delivered to the patient's nose at high flow rates $\left(2-60 \mathrm{~L} \cdot \mathrm{min}^{-1}\right)$ [1]. NHF can assist ventilation and prevent intubation in both adults and children with respiratory failure [2-5]. Several mechanisms have been proposed to explain the responses observed with the use of this therapy. NHF could lead to improvements in respiratory mechanics [6], better humidification of airways [7], reductions in anatomical dead space [8,9], increases in end-expiratory lung volume [10-12] and decreases in ventilatory demand due to a reduction in work of breathing [4, 9, 13-15]. The decrease in ventilatory demand has been attributed to washout of the anatomical nasal dead space [8]; however, dynamic measures of dead space volume and carbon dioxide $\left(\mathrm{CO}_{2}\right)$ production during tidal breathing were not performed, leaving the actual mechanism of action for the effects produced by NHF still unknown.

Sleep is a unique opportunity to study physiological mechanisms of NHF as we can avoid potential conscious confounders. We have previously shown that ventilatory responses to NHF are different during wakefulness and sleep [16]. These differences may explain the heterogeneous responses reported when examining mechanisms of NHF in the clinical setting. In fact, when controlled for sleep, we observed a more homogeneous response to NHF. Both minute ventilation and work of breathing decrease, while $\mathrm{CO}_{2}$ levels remain constant [9]. The reduction in ventilation is possibly due to a decrease in dead space ventilation due to nasal dead space washout $[8,17]$. However, by decreasing work of breathing, NHF can also decrease $\mathrm{CO}_{2}$ production and, therefore, reduce the amount of minute ventilation required to maintain constant levels of $\mathrm{CO}_{2}$.

To determine whether the reduction in minute ventilation is driven by the decrease in work of breathing or by a reduction in dead space ventilation, we measured the effects of $\mathrm{NHF}$ on $\mathrm{CO}_{2}$ production during sleep in controls and chronic obstructive pulmonary disease (COPD) patients. Specifically, using a metabolic hood and standard polysomnography with $\mathrm{CO}_{2}$ monitoring, we measured $\mathrm{CO}_{2}$ production and calculated alveolar ventilation with and without the use of NHF during stable sleep. We hypothesised that NHF would reduce dead space ventilation and that the amount of the reduction would depend on the extent of dead space. We also hypothesised that, in contrast to controls, COPD patients would show a reduction in $\mathrm{CO}_{2}$ production, thereby also reducing alveolar ventilation.

\section{Methods}

Participants were recruited from the Johns Hopkins pulmonary clinics and surrounding community in the USA. Subjects $>18$ years old and consenting to participate were included. Exclusion criteria were exacerbation of COPD or severe illnesses within 8 weeks prior to the study, severe heart and liver disease, home use of oxygen $\left(\mathrm{O}_{2}\right)$, and/or chronic use of opioids, benzodiazepines or other sedatives.

In a previous study [9], we detected a 30-40\% reduction in minute ventilation, work of breathing and tidal volume with the use of NHF. As we planned to investigate whether changes in metabolic rate contributed to minute ventilation reduction, we estimated that 10 subjects would be required to reproduce the previously observed effect on ventilation and to study the contribution of variation in metabolic rate.

The study was approved by the Johns Hopkins Medical Institution Human Investigation Review Board.

\section{Equipment setup}

Subjects enrolled underwent an overnight sleep study under a metabolic hood to measure $\mathrm{CO}_{2}$ production while using NHF. During the study, we collected standard polysomnography signals including transcutaneous $\mathrm{CO}_{2}$ (TCM3; Radiometer Medical, Brønshøj, Denmark). Ventilation was monitored with respiratory inductive plethysmography (Respitrace; AMI, Ardsley, NY, USA). At the beginning of the protocol, subjects wore a face mask attached to a pneumotachograph [18] and end-tidal $\mathrm{CO}_{2}$ monitor (VacuMed, Ventura, CA, USA). Subjects breathed through the mask for $\sim 30 \mathrm{~s}$ to calibrate the respiratory inductive plethysmography with the integrated pneumotachograph signal. Additionally, participants performed a slow exhalation manoeuvre to elicit an accurate end-tidal $\mathrm{CO}_{2}$ reading. We scaled the transcutaneous $\mathrm{CO}_{2}$ measurement to the obtained end-tidal $\mathrm{CO}_{2}$ values. After calibration procedures, the mask was removed and subjects wore only a nasal cannula for the intermittent delivery of NHF.

Once participants were fully monitored and comfortable in bed, we placed a metabolic hood (Quark RMR; Cosmed, Rome, Italy) covering their head and chest to collect the air exhaled by the subject (figure 1). This system allows the measurement of $\mathrm{O}_{2}$ consumption and $\mathrm{CO}_{2}$ production in an individual without wearing a mask. The long sheet attached to the hood sits around the upper part of the subject's body, avoiding air leaks. A pump continuously aspirates air from the hood to the analysis equipment, directing the exhaled air towards the hood exit and away from other potential leak points. The exhaled air is collected and analysed to compute $\mathrm{CO}_{2}$ production, $\mathrm{O}_{2}$ consumption and energy expenditure. 


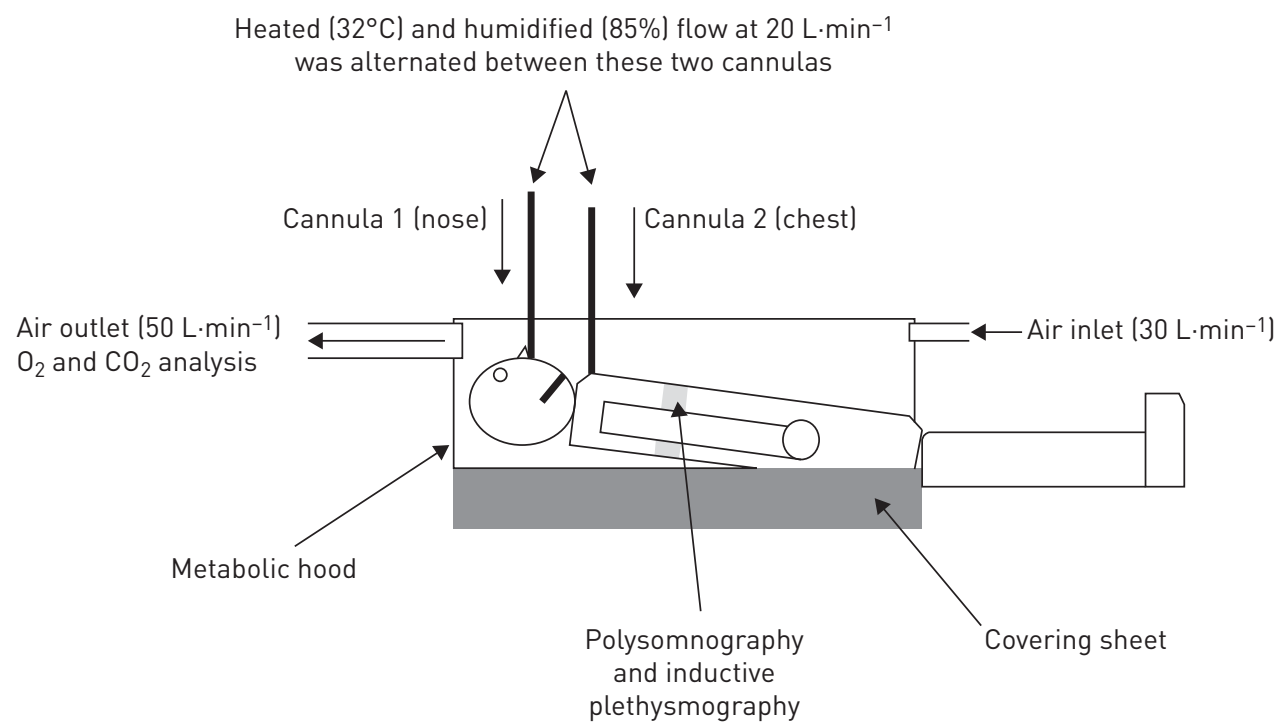

FIGURE 1 Schematic representation of the experimental setup. Subjects underwent a polysomnography study in which we monitored the ECG, electro-oculogram, electroencephalogram, electromyogram, pulse oximetry and transcutaneous carbon dioxide $\left(\mathrm{CO}_{2}\right)$. Ventilation was monitored with respiratory inductive plethysmography, calibrated with a flow sensor at the beginning of the experiment. Once subjects were monitored, we placed a metabolic hood covering the subject's upper body. The system consisted of a rigid plastic transparent cover attached to a plastic sheet, which sat around the subject's upper body. The sheet created a high-resistance area around the subject, avoiding air leaks. Additionally, a pump aspirated air from inside the hood, directing the flow to the analysis equipment and away from other potential leak points. Air could enter into the hood through the air inlet or through the system for nasal high flow (NHF) delivery. We maintained a high aspirating flow from the hood to minimise leaks. Oxygen $\left(\mathrm{O}_{2}\right)$ consumption and $\mathrm{CO}_{2}$ production were measured at the outlet flow. Participants wore a nasal cannula (cannula 1) for the intermittent delivery of high-flow therapy $\left(20 \mathrm{~L} \cdot \mathrm{min}^{-1}, 85 \%\right.$ humidity, $\left.32^{\circ} \mathrm{C}\right)$. An additional cannula was placed in the subject's chest (cannula 2) for delivery of the high flow whenever the NHF was turned off. Therefore, we maintained a constant flow to the hood interior.

In our setting, we sought to determine $\mathrm{CO}_{2}$ production and energy expenditure during the NHF therapy, which would alter the flow delivered to the interior of the hood. Therefore, a high flow rate at the pump collecting air from the hood $\left(50 \mathrm{~L} \cdot \mathrm{min}^{-1}\right)$ was maintained to avoid leaks and to ensure constant experimental conditions. The incoming flow to the hood was the sum of the NHF flow $\left(20 \mathrm{~L} \cdot \mathrm{min}^{-1}\right)$ and a room air inlet flow $\left(30 \mathrm{~L} \cdot \mathrm{min}^{-1}\right)$. Whenever the NHF to the subject's nose was turned off, we initiated the delivery of an equal flow to the subject's chest via a secondary cannula, maintaining the overall flow to the interior of the hood constant (figure 1). As it was important to maintain a constant flow to the metabolic hood, we performed a series of pilot experiments using a constant source of $\mathrm{CO}_{2}$ to ensure that alternating NHF would not interfere with metabolic measurements.

\section{Study protocol}

Subjects were admitted to the sleep centre at approximately 19:00 h to get accustomed to the experimental procedures and to standardise their level of activity prior to the sleep studies. Once participants were fully monitored, we started the delivery of a flow of $20 \mathrm{~L} \cdot \mathrm{min}^{-1}, 85 \%$ humidity and $32^{\circ} \mathrm{C}$ through a nasal cannula during wakefulness for acclimatisation. We used a modified S8 continuous positive airway pressure machine (ResMed, Bella Vista, Australia) that was able to generate constant flows up to $30 \mathrm{~L} \cdot \mathrm{min}^{-1}$. The device was also designed with a special hose with active heating that maintained warm and humid delivered air. Between 22:00 and 23:00 h, subjects initiated sleep with NHF on at $20 \mathrm{~L} \cdot \mathrm{min}^{-1}$. When subjects were in stable non-rapid eye movement (NREM) stage 2 sleep, we alternated periods of NHF on/off at 5- to 10-min intervals (figure 2). During the periods when NHF was turned off, the flow to the second cannula (to the subject's chest) was turned on at the same rate, humidity and temperature of the NHF. For each subject, pairs of NHF on/off trials were selected when intervals were absent of interruptions in sleep and there was no significant reduction in pulse wave amplitude of $>50 \%$ from baseline as a marker for cortical activity or skin nerve activity. On average, three pairs of NHF on/off trials for each subject were obtained. Measurements were summarised to produce one mean value of "NHF off" and "NHF on" for each subject, which were used for the statistical comparisons. 


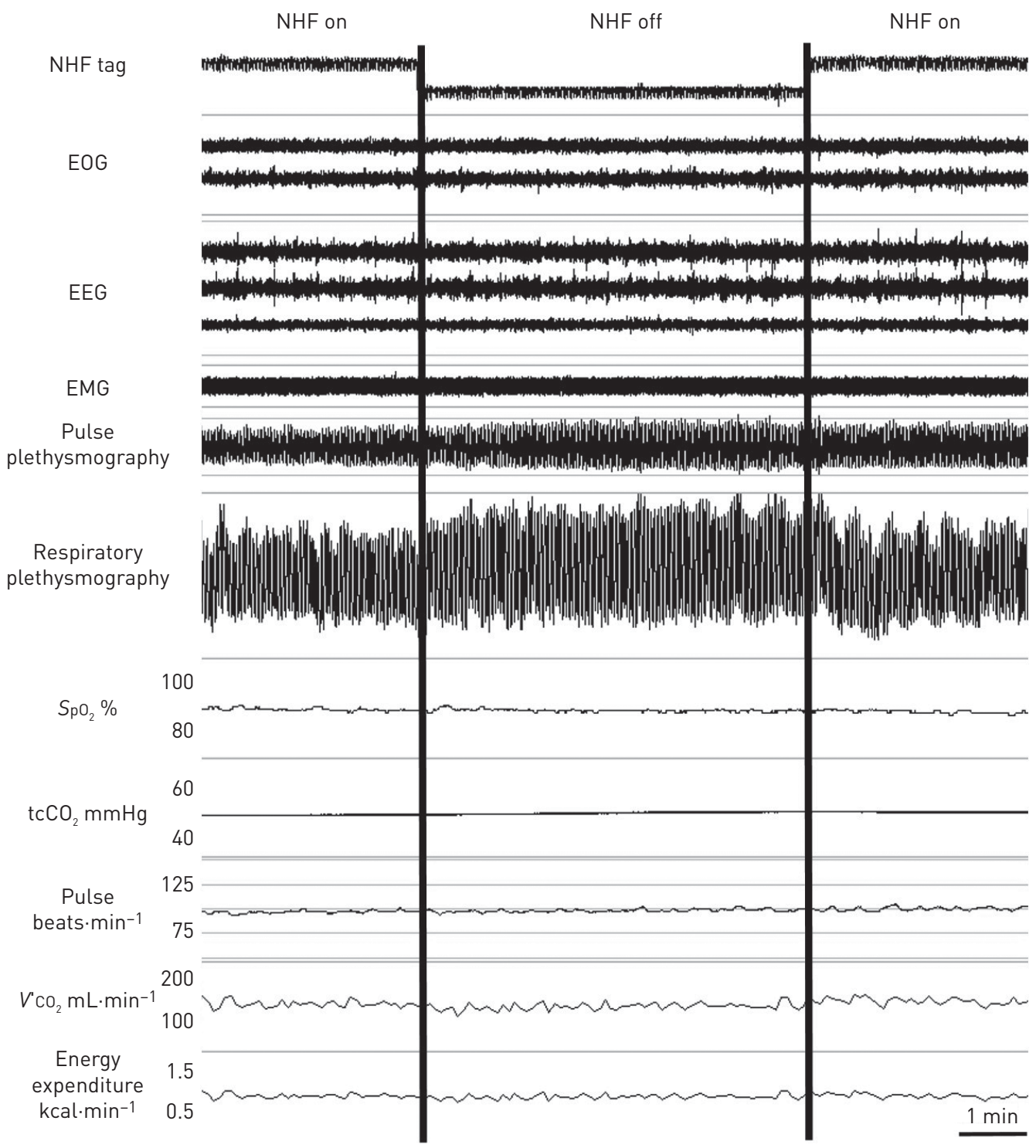

FIGURE 2 Experimental protocol. NHF: nasal high flow; EOG: electro-oculogram; EMG: electromyogram; $\mathrm{SpO}_{2}$ : arterial oxygen saturation measured by pulse oximetry; $\mathrm{CO}_{2}$ : carbon dioxide; tc $\mathrm{CO}_{2}$ : transcutaneous $\mathrm{CO}_{2}$; $V^{\prime} \mathrm{CO}_{2}: \mathrm{CO}_{2}$ production. Participants slept under $\mathrm{NHF}\left(20 \mathrm{~L} \cdot \mathrm{min}^{-1}, 85 \%\right.$ humidity, $\left.32^{\circ} \mathrm{C}\right)$ for acclimatisation. Once achieving non-rapid eye movement stage 2 sleep, we alternated NHF delivery (NHF on) with no flow delivered (NHF off) to the nose. During the NHF off period, we delivered the same flow to the subject's chest to maintain constant flow delivery to the hood interior. We selected periods without arousals or changes in sympathetic activity. During periods of NHF on, we could observe a reduction in ventilation, but no change in $\mathrm{CO}_{2}$ production or energy expenditure.

\section{Parameters calculation and statistical analysis}

Data were recorded using a RemLogic polysomnography recording station (Natus, Pleasanton, CA, USA) and transferred to IgorPro data analysis software (WaveMetrics, Lake Oswego, OR, USA). We derived minute ventilation, respiratory rate and tidal volume from the calibrated respiratory inductive plethysmography signal. Physiological dead space ventilation was calculated using the equation for alveolar ventilation: $\mathrm{PaCO}_{2}=0.863 \times V^{\prime} \mathrm{CO}_{2} /\left(V^{\prime} \mathrm{E}-V^{\prime} \mathrm{D}\right.$ ), where $\mathrm{PaCO}_{2}$ is arterial $\mathrm{CO}_{2}$ tension (for which calibrated transcutaneous $\mathrm{CO}_{2}$ measurements were used), $V^{\prime} \mathrm{CO}_{2}$ is $\mathrm{CO}_{2}$ production (measured with the metabolic hood), $V^{\prime} \mathrm{E}$ is minute ventilation and $V^{\prime} \mathrm{D}$ is the physiological dead space ventilation per minute [19].

Tidal dead space volume was calculated by dividing dead space ventilation by respiratory rate. Dead space fraction was computed as dead space volume divided by tidal volume. Anatomical dead space volume was estimated from participant's height, as previously described [20-22].

Measurements with and without NHF were compared by the paired t-test. The degree of response in controls and COPD patients was compared by the nonpaired t-test. The correlation of changes in dead 
space to baseline dead space was analysed using Pearson correlation coefficient. We considered p-values $<0.05$ as statistically significant.

\section{Results}

12 participants were recruited (six controls and six COPD patients), but one subject (with COPD) could not sleep under the metabolic hood and was excluded. Table 1 shows the demographics of the population enrolled.

We detected an awakening in 12 out of the $98 \mathrm{NHF}$ on/off trials, some of them apparently induced by the transition of the state of NHF (on to off or vice versa). Once an arousal was detected, we waited longer periods until subjects reached stable sleep or excluded the analysis on that segment if sleep was not resumed.

\section{Effect of NHF on respiratory pattern during sleep under a metabolic hood}

In figure 2, we show the effect of the NHF on and off conditions on ventilation during sleep in one individual (COPD1). It can be seen that our experimental setup of turning NHF on and off during sleep (see "NHF tag" trace) did not disturb sleep continuity (see electro-oculogram, EEG and electromyogram traces) or lead to an activation of autonomic nerve activity, as illustrated by the stability of the heart rate (pulse) and pulse wave amplitude in this patient. The effects of NHF on ventilation are illustrated by comparing the tidal volume signal (see respiratory plethysmography trace) with the $\mathrm{O}_{2}$ saturation, transcutaneous $\mathrm{CO}_{2}, \mathrm{CO}_{2}$ production and energy expenditure. While tidal volumes increased during the NHF off condition, there was no change in transcutaneous $\mathrm{CO}_{2}, \mathrm{O}_{2}$ saturation, $\mathrm{CO}_{2}$ production or energy expenditure, indicating that alveolar ventilation did not change.

\section{Effect of NHF on ventilation}

Pooled data are shown figure 3. At baseline, compared with controls, COPD patients had a slightly but not statistically significant higher minute ventilation (controls $4.9 \pm 0.5 \mathrm{~L} \cdot \mathrm{min}^{-1}$ versus COPD patients $6.4 \pm 0.6 \mathrm{~L} \cdot \mathrm{min}^{-1}$; not significant), respiratory rate (controls $15.8 \pm 1.0$ breaths $\cdot \mathrm{min}^{-1}$ versus COPD patients $18.4 \pm 1.3$ breaths $\cdot \mathrm{min}^{-1}$; not significant) and dead space ventilation (controls $1.8 \pm 0.3 \mathrm{~L} \cdot \mathrm{min}^{-1}$ versus COPD patients $3.2 \pm 0.6 \mathrm{~L} \cdot \mathrm{min}^{-1}$; not significant).

In response to NHF, we noticed a significant reduction in minute ventilation in all subjects, as shown in figure 3a (NHF off $5.6 \pm 0.4 \mathrm{~L} \cdot \mathrm{min}^{-1}$ versus $\mathrm{NHF}$ on $4.8 \pm 0.4 \mathrm{~L} \cdot \mathrm{min}^{-1} ; \mathrm{p}<0.05$ ). The reduction in ventilation was associated with a reduction in tidal volumes (figure $3 \mathrm{~b})$ from NHF off $(340 \pm 30 \mathrm{~mL})$ to NHF on

\section{TABLE 1 Characteristics of the participants}

\section{Controls}

6

\section{Subjects \\ Demographics}

Age years

Sex

Male

Female

Weight kg

Height $\mathrm{cm}$

BMI $\mathrm{kg} \cdot \mathrm{m}^{-2}$

Lung function

$\mathrm{FEV}_{1} / \mathrm{FVC} \%$

FEV1 \% pred

Baseline values

Pulse beats $\min ^{-1}$

$\mathrm{SpO}_{2} \%$

$\mathrm{ETCO}_{2} \mathrm{mmHg}$
$44.2 \pm 18.4$

2

4

$70.3 \pm 15.4$

$164.8 \pm 12.4$

$25.3 \pm 6.0$

$83.5 \pm 14.9$

$86.8 \pm 15.4$

$69.3 \pm 11.1$

$95.2 \pm 2.3$

$40.9 \pm 2.1$
COPD patients

Data are presented as $\mathrm{n}$ or mean $\pm \mathrm{SD}$. COPD: chronic obstructive pulmonary disease; BMI: body mass index; $\mathrm{FEV}_{1}$ : forced expiratory volume in $1 \mathrm{~s}$; $\mathrm{FVC}$ : forced vital capacity; $\mathrm{SpO}_{2}$ : arterial oxygen saturation measured by pulse oximetry; $\mathrm{ETCO}_{2}$ : end-tidal carbon dioxide. We enrolled six controls and five COPD patients. Our sample consisted of more females in both groups. Patients with COPD were older, but with similar BMI. $\mathrm{FEV}_{1} \%$ pred was lower for COPD patients, as determined by enrolment, but there were no differences in $\mathrm{SpO}_{2}$ and $\mathrm{ETCO}_{2}$ during baseline measurements at the beginning of the protocol. 

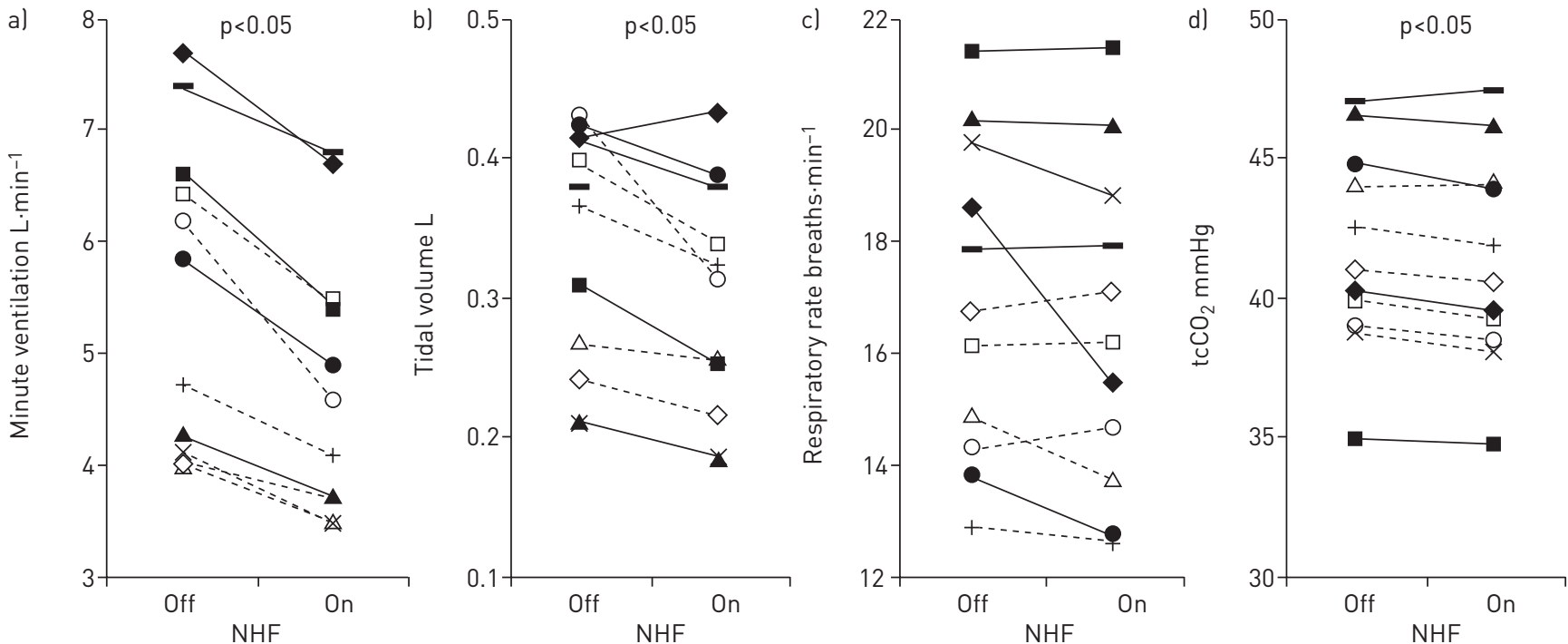

....- Controls $(0, \square, \diamond, \triangle,+, \times) ;-\operatorname{COPD}$ patients $(\bullet, \mathbf{\square}, \diamond, \mathbf{\Delta}, \mathbf{-})$

FIGURE 3 Ventilatory response to nasal high flow (NHF) in controls and chronic obstructive pulmonary disease (COPD) patients: a) minute ventilation, b) tidal volume, c) respiratory rate and d) transcutaneous carbon dioxide $\left(\mathrm{tc} \mathrm{CO}_{2}\right)$. The plots display average individual values for each parameter under the two conditions (NHF off/NHF on $\left(20 \mathrm{~L} \cdot \mathrm{min}^{-1}\right)$ ). Individual controls and COPD patients are identified by different symbols (the same coding system for subjects is used throughout figures 3-5). We observed a significant reduction in minute ventilation for all subjects (NHF off $5.6 \pm 0.4 \mathrm{~L} \cdot \mathrm{min}^{-1}$ versus NHF on $4.8 \pm 0.4 \mathrm{~L} \cdot \mathrm{min}^{-1}$ ] with the use of NHF. Tidal volumes were also significantly reduced by the use of NHF in all but one subject (NHF off $340 \pm 30 \mathrm{~mL}$ versus NHF on $300 \pm 30 \mathrm{~mL}$ ). Overall, respiratory rate did not change significantly, but it is noteworthy to observe a reduction in respiratory rate for the one individual with no reduction in tidal volume. We noticed a slight, but statistically significant, reduction in $\mathrm{tcCO}_{2}$ with the use of NHF (NHF off $42.6 \pm 1.2 \mathrm{mmHg}$ versus NHF on $42.1 \pm 1.2 \mathrm{mmHg}$ ).

$(300 \pm 30 \mathrm{~mL} ; \mathrm{p}<0.05)$. There was no significant change in respiratory rates (figure $3 \mathrm{c}$ ) and a minimal reduction in transcutaneous $\mathrm{CO}_{2}$ (figure 3d), which fell from NHF off $(41.7 \pm 1.1 \mathrm{mmHg}$ ) to $\mathrm{NHF}$ on $(41.3 \pm 1.2 \mathrm{mmHg} ; \mathrm{p}<0.05)$. Changes in these parameters in response to NHF were similar for controls and COPD patients. Of note, there was one participant who had moderate COPD (female, 55 years, forced expiratory volume in $1 \mathrm{~s} 38 \%$ of predicted) who reduced minute ventilation from NHF off $\left(7.7 \mathrm{~L} \cdot \mathrm{min}^{-1}\right)$ to NHF on $\left(6.7 \mathrm{~L} \cdot \mathrm{min}^{-1}\right)$ due to a $17 \%$ decrease in respiratory rate from 18.6 to 15.5 breaths $\mathrm{min}^{-1}$ without a significant change in her tidal volume (from 413 to $433 \mathrm{~mL}$ ).

In figure $4 \mathrm{a}$, we show pooled data for alveolar ventilation which remained unchanged in controls and COPD subjects. However, there was a substantial $(\sim 40 \%)$ reduction in dead space ventilation (NHF off $2.5 \pm 0.4 \mathrm{~L} \cdot \mathrm{min}^{-1}$ versus $\mathrm{NHF}$ on $1.6 \pm 0.4 \mathrm{~L} \cdot \mathrm{min}^{-1} ; \mathrm{p}<0.05$; figure $4 \mathrm{~b}$ ), dead space fraction (NHF off $0.42 \pm 0.03$ versus NHF on $0.31 \pm 0.05 ; \mathrm{p}<0.05$; figure $4 \mathrm{c}$ ) and dead space volume (NHF off $144 \pm 19 \mathrm{~mL}$ versus NHF on $98 \pm 22 \mathrm{~mL}$; $<<0.05$; figure $4 \mathrm{~d}$ ) with the use of NHF. The reduction in dead space ventilation was similar for both controls and COPD patients. Of note, the individual with moderate COPD who reduced the respiratory rate from 18.6 to 15.5 breaths $\cdot \mathrm{min}^{-1}$ on NHF, as mentioned previously, did not have a significant change in either alveolar ventilation (NHF off $3.3 \mathrm{~L} \cdot \mathrm{min}^{-1}$ versus NHF on $3.4 \mathrm{~L} \cdot \mathrm{min}^{-1}$ ) or transcutaneous $\mathrm{CO}_{2}$ (NHF off $40.3 \mathrm{mmHg}$ versus NHF on $39.5 \mathrm{mmHg}$ ). However, she had a substantial reduction in dead space ventilation $\left(1.1 \mathrm{~L} \cdot \mathrm{min}^{-1}\right)$ that was due to both the reduction in respiratory rate and a reduction in tidal dead space volume (NHF off $236 \mathrm{~mL}$ versus NHF on $214 \mathrm{~mL}$ ).

\section{Determinants of reductions in dead space ventilation}

There was no correlation between baseline respiratory rate, minute ventilation or anatomical dead space volume with either the reduction in dead space ventilation or the reduction in tidal dead space volume. In contrast, the degree of physiological dead space at baseline correlated with the reduction of dead space ventilation with NHF: the greater the dead space fraction of an individual at baseline, the greater the reduction in dead space ventilation with NHF. As can be seen in figure 5 , for each $10 \%$ increase in dead space fraction there is a $208 \mathrm{~mL} \cdot \mathrm{min}^{-1}$ reduction in dead space ventilation with the use of $\mathrm{NHF}\left(\mathrm{r}^{2}=0.36 ; \mathrm{p}<0.05\right)$.

\section{Discussion}

In the present work, we evaluated the effects of the use of NHF during sleep on ventilation and directly measured $\mathrm{CO}_{2}$ production using a metabolic hood and polysomnography. First, we observed that responses in ventilation to NHF during sleep were similar in COPD patients and controls. Second, the use 

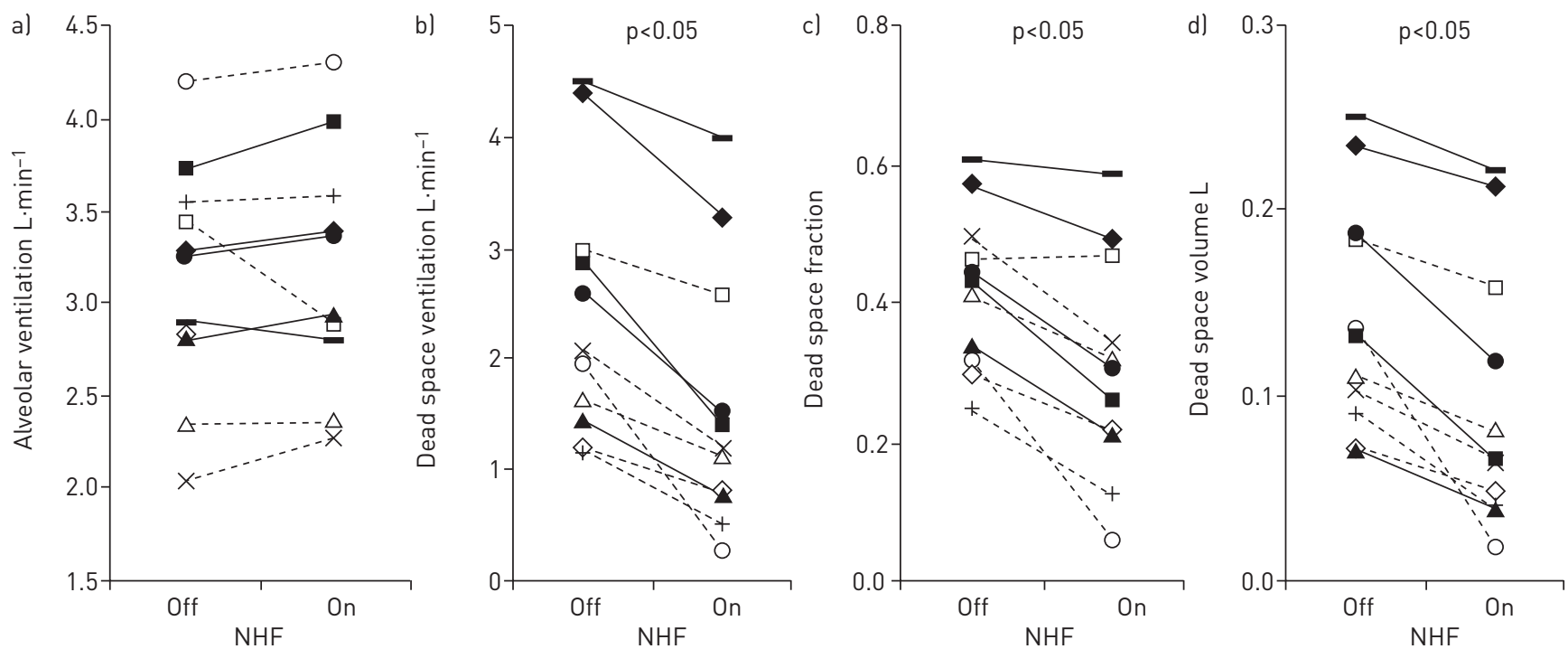

-...- Controls $(0, \square, \diamond, \Delta,+, x) ;-\operatorname{COPD}$ patients $(\bullet, \mathbf{\bullet}, \diamond, \mathbf{\Delta}, \mathbf{-})$

FIGURE 4 Alveolar ventilation and dead space with nasal high flow (NHF) in controls and chronic obstructive pulmonary disease (COPD) patients: a) alveolar ventilation, b) dead space ventilation, c) dead space fraction (dead space volume/tidal volume) and d) dead space volume. The plots display average individual values for each parameter under the two conditions (NHF off/NHF on ( $\left.20 \mathrm{~L} \cdot \mathrm{min}^{-1}\right)$ ). Individual controls and COPD patients are identified by different symbols (the same coding system for subjects is used throughout figures 3-5). There was no significant change in alveolar ventilation with the use of NHF. However, in all subjects NHF produced a significant reduction in dead space ventilation (NHF off $2.5 \pm 0.4 \mathrm{~L} \cdot \mathrm{min}^{-1}$ to $1.7 \pm 0.3 \mathrm{~L} \cdot \mathrm{min}^{-1}$ ), dead space fraction (NHF off $0.43 \pm 0.03$ to NHF on $0.33 \pm 0.04$ ) and dead space volume (NHF off $150 \pm 20 \mathrm{~mL}$ to NHF on $100 \pm 20 \mathrm{~mL}$ ).

of NHF led to a substantial decrease in minute ventilation due to a reduction in dead space ventilation without a significant change in alveolar ventilation, $\mathrm{CO}_{2}$ production, energy expenditure or transcutaneous $\mathrm{CO}_{2}$. Third, the reduction in dead space ventilation depended on the physiological dead space fraction and not anatomical dead space volume, respiratory rate or minute ventilation at baseline NHF off. Although we did not observe a reduction in either alveolar ventilation or $\mathrm{CO}_{2}$ production during our short trials of NHF, it is still possible that longer trials of NHF may also affect these parameters.

Several previous studies have demonstrated a washout of the anatomical dead space with NHF using imaging techniques in upper airway models [8] or the human upper airway in volunteers [17]. These

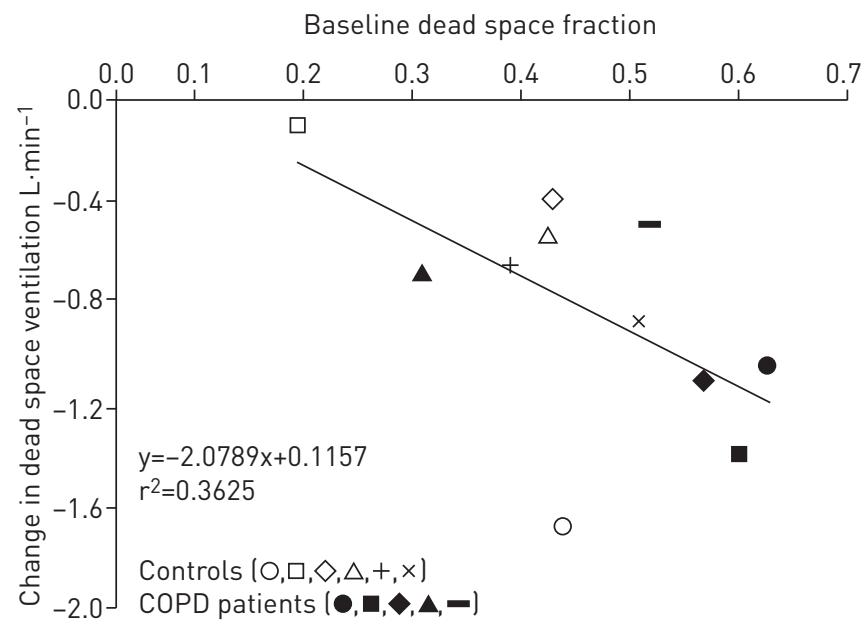

FIGURE 5 Dead space fraction (dead space volume/tidal volume) correlation. The data points represent individual averages for controls and chronic obstructive pulmonary disease (COPD) patients. Individual controls and COPD patients are identified by different symbols (the same coding system for subjects is used throughout figures 3-5). We noticed an inverse correlation of the amount of the reduction nasal high flow (NHF) produced in dead space ventilation with the values of baseline dead space fraction (measured with NHF off). 
studies, however, could not elucidate the effect of NHF on dead space clearance because of technical restrictions, and did not evaluate $\mathrm{CO}_{2}$ production and metabolism [17]. We were able to show that baseline physiological dead space volume is related to the amount of dead space washout at aiven flow rate. Physiological dead space is known to directly correlate with tidal volume [23]. We now show that the lower the tidal dead space fraction, the lower the reduction in dead space ventilation with NHF. Thus, it appears that individuals with a higher tidal volume due to increases in the dead space fraction benefit more from the use of NHF compared with those with a lower dead space fraction.

NHF has been extensively used for treating respiratory failure in different clinical settings. The warming and humidification of the air allow the delivery of higher flows, creating a low level of positive end-expiratory pressure and increasing end-expiratory lung volume [10-12]. Furthermore, NHF reduces minute ventilation during both sleep and wakefulness, without increasing $\mathrm{CO}_{2}$ levels $[9,16,24,25]$. The respiratory pattern response to NHF apparently differs when individuals are awake or sleeping [16], thus explaining potential differences in respiratory rate responses between individuals [16]. To exclude this confounder, we determined ventilatory responses during sleep, and showed that NHF reduced minute ventilation and dead space ventilation similarly in controls and patients with mild to moderate COPD. Moreover, the reduction in dead space ventilation was associated with no change in alveolar ventilation, $\mathrm{CO}_{2}$ production or transcutaneous $\mathrm{CO}_{2}$. This is in contrast to previous studies, in which we and others have shown a significant reduction in work of breathing following the reduction in minute ventilation [4, 9, 13-15].

There are several explanations for these discrepancies. First, it is possible that our on/off trials of NHF were too short to translate into changes in $\mathrm{CO}_{2}$ production and energy expenditure. Second, during sleep, energy expenditure is low and for individuals with a low amount of ventilation, we expect a low level of energy expenditure related to respiratory muscle activity. Thus, changes in work of breathing could have been too small to be detected by measurements of energy expenditure. Third, ventilatory responses to NHF were similar in controls and COPD patients, which suggests that breathing mechanics were not markedly different between these groups. Additionally, the number of patients enrolled was too small to exclude minor differences between the responses in COPD patients and controls. We also could not compare the differential responses between sexes. The complexity of the experimental design prevented the enrolment of a larger sample. Furthermore, our patients did not have severe obstruction. Patients with more severe COPD disease may have shown a differential response to NHF.

There are also several strengths in our study. First, this study performed on/off experiments in a very controlled setting without influencing the variables of the metabolic hood. Second, polysomnography was used to control for biological variables related to changes in sleep stages, movements and conscious influences. As a consequence, we obtained data from stable NREM stage 2-3 sleep in which breathing pattern and metabolism are very regular, allowing the detection of even small changes in $\mathrm{CO}_{2}$ production. Third, we quantified ventilation with calibrated respiratory inductive plethysmography, which is devoid of any artificial changes in ventilation that may arise with the use of either nasal or face masks. Taken together, our data indicate that the immediate ventilatory responses to NHF are due to a reduction in dead space ventilation. Whether these changes translate into reductions in energy expenditure may depend on NHF being used over prolonged periods of time or in patients with high ventilatory demands.

Our study has immediate clinical implications. First, we demonstrate that alveolar ventilation remains constant during the use of NHF and the driver for the reduction in minute ventilation is a reduction in dead space ventilation. Therefore, NHF could be particularly useful in patients with large dead space ventilation, maximising its potential for unloading the respiratory system. Second, we now provide an explanation for the heterogeneous response to NHF observed in previous studies. While some individuals lowered their arterial $\mathrm{CO}_{2}$ in response to NHF, others did lower their respiratory rate [24]. We hypothesise that individuals with higher physiological dead space ventilation showed a reduction in arterial $\mathrm{CO}_{2}$. Finally, although $\mathrm{CO}_{2}$ production remained constant during our short on/off trials of NHF, it is possible that longer use of NHF would lead to reductions in work of breathing and $\mathrm{CO}_{2}$ production, and could prevent nocturnal hypercapnia. However, studies with longer exposure to NHF are required to further evaluate these hypotheses.

In conclusion, we show that the mechanism for minute ventilation reduction during short-term use of NHF during sleep is not related to a reduction in $\mathrm{CO}_{2}$ production. Rather, it is caused by a reduction in dead space ventilation. The degree of the reduction in minute ventilation is correlated to the amount of baseline physiological dead space, measured as the dead space fraction. Therefore, NHF could be used to unload the respiratory system, particularly in patients with a very high dead space fraction. 
Conflict of interest: P. Biselli reports grants from the National Institutes of Health (NIH) (HL105546), and grants from the Brazilian funding agencies FAPESP and $\mathrm{CNPq}$, during the conduct of the study. K. Fricke reports grants from the NIH (HL105546), during the conduct of the study. L. Grote reports grants from ResMed Foundation and Philips Foundation, grants, personal fees and nonfinancial support from ResMed (speaker's bureau and study support), personal fees from Philips (speaker's bureau), personal fees from Itamar (speaker's bureau and study support), and personal fees from Weinmann (for consultancy), outside the submitted work. A.T. Braun reports grants from the NIH (HL105546), during the conduct of the study. J. Kirkness has received grants from the NIH (HL105546), and is director of clinical affairs for Fisher \& Paykel Healthcare, during the conduct and publication of the study. P. Smith reports grants from the NIH (HL105546), during the conduct of the study. A. Schwartz reports grants from the NIH (HL105546), during the conduct of the study. H. Schneider reports grants from the NIH (HL105546), personal fees for consulting and nonfinancial support with devices in patients with CF from Fisher \& Paykel Healthcare, grants from ResMed (for sponsored research on the effect of NHF in COPD), and personal fees from TNI Medical (for consultancy; high flow in COPD), during the conduct of the study; personal fees for consultancy from Fisher \& Paykel Healthcare and TNI Medical, outside the submitted work; in addition, $\mathrm{H}$. Schneider has a US patent: 7,080,645 issued to TNI Medical (anti-snoring device, method for reducing snoring, and a nasal air cannula).

Support statement: This study was funded by the Ministério da Ciência, Tecnologia e Inovação, Conselho Nacional de Desenvolvimento Científico e Tecnológico (grant 200817/2012-4), Fundação de Amparo à Pesquisa do Estado de São Paulo (grant 2012/05190-0) and the US Dept of Health and Human Services, National Institutes of Health, National Heart, Lung, and Blood Institute (grant 105546). Funding information for this article has been deposited with the Crossref Funder Registry.

\section{References}

$1 \quad$ Nishimura M. High-flow nasal cannula oxygen therapy in adults. J Intensive Care 2015; 3: 15.

2 Frat J-P, Thille AW, Mercat A, et al. High-flow oxygen through nasal cannula in acute hypoxemic respiratory failure. N Engl J Med 2015; 372: 2185-2196.

3 Schibler A, Pham TMT, Dunster KR, et al. Reduced intubation rates for infants after introduction of high-flow nasal prong oxygen delivery. Intensive Care Med 2011; 37: 847-852.

4 Saslow JG, Aghai ZH, Nakhla TA, et al. Work of breathing using high-flow nasal cannula in preterm infants. J Perinatol 2006; 26: 476-480.

5 Maggiore SM, Idone FA, Vaschetto R, et al. Nasal high-flow versus Venturi mask oxygen therapy after extubation. effects on oxygenation, comfort, and clinical outcome. Am J Respir Crit Care Med 2014; 190: 282-288.

6 Dysart K, Miller TL, Wolfson MR, et al. Research in high flow therapy: mechanisms of action. Respir Med 2009; 103: $1400-1405$.

7 Rea H, McAuley S, Jayaram L, et al. The clinical utility of long-term humidification therapy in chronic airway disease. Respir Med 2010; 104: 525-533.

8 Möller W, Celik G, Feng S, et al. Nasal high flow clears anatomical dead space in upper airway models. J Appl Physiol 2015; 118: 1525-1532.

9 Biselli PJC, Kirkness JP, Grote L, et al. Nasal high-flow therapy reduces work of breathing compared with oxygen during sleep in COPD and smoking controls: a prospective observational study. J Appl Physiol 2017; 122: 82-88.

10 Groves N, Tobin A. High flow nasal oxygen generates positive airway pressure in adult volunteers. Aust Crit Care 2007; 20: 126-131.

11 Corley A, Caruana LR, Barnett AG, et al. Oxygen delivery through high-flow nasal cannulae increase end-expiratory lung volume and reduce respiratory rate in post-cardiac surgical patients. Br J Anaesth 2011; 107: 998-1004.

12 McGinley B, Halbower A, Schwartz AR, et al. Effect of a high-flow open nasal cannula system on obstructive sleep apnea in children. Pediatrics 2009; 124: 179-188.

13 Pham TMT, O'Malley L, Mayfield S, et al. The effect of high flow nasal cannula therapy on the work of breathing in infants with bronchiolitis. Pediatr Pulmonol 2015; 50: 713-720.

14 Mauri T, Turrini C, Eronia N, et al. Physiologic effects of high-flow nasal cannula in acute hypoxemic respiratory failure. Am J Respir Crit Care Med 2017; 195: 1207-1215.

15 Vargas F, Saint-Leger M, Boyer A, et al. Physiologic effects of high-flow nasal cannula oxygen in critical care subjects. Respir Care 2015; 60: 1369-1376.

16 Mundel T, Feng S, Tatkov S, et al. Mechanisms of nasal high flow on ventilation during wakefulness and sleep. J Appl Physiol 2013; 114: 1058-1065.

17 Möller W, Feng S, Domanski U, et al. Nasal high flow reduces dead space. J Appl Physiol 2017; 122: 191-197.

18 Kirkness JP, Verma M, McGinley BM, et al. Pitot-tube flowmeter for quantification of airflow during sleep. Physiol Meas 2011; 32: 223-237.

19 Hubmayr RD. Setting the ventilator. In: Tobin MJ, ed. Principles and Practice of Mechanical Ventilation. 1st Edn. New York, McGraw-Hill, 1994; pp. 191-206.

20 Schneider H, Krishnan V, Pichard LE, et al. Inspiratory duty cycle responses to flow limitation predict nocturnal hypoventilation. Eur Respir J 2009; 33: 1068-1076.

21 Kars AH, Bogaard JM, Stijnen T, et al. Dead space and slope indices from the expiratory carbon dioxide tension-volume curve. Eur Respir J 1997; 10: 1829-1836.

22 Fowler W, Comroe J Jr. Lung function studies. I. The rate of increase of arterial oxygen saturation during the inhalation of 100 per cent $\mathrm{O}_{2}$. J Clin Invest 1948; 27: 327-334.

23 Chakrabarti M, Gordon G, Whitwam J. Relationship between tidal volume and deadspace during high frequency ventilation. Br J Anaesth 1986; 58: 11-17.

24 Nilius G, Franke KJ, Domanski U, et al. Effects of nasal insufflation on arterial gas exchange and breathing pattern in patients with chronic obstructive pulmonary disease and hypercapnic respiratory failure. Adv Exp Med Biol 2013; 755: 27-34.

25 Fricke K, Tatkov S, Domanski U, et al. Nasal high flow reduces hypercapnia by clearance of anatomical dead space in a COPD patient. Respir Med Case Rep 2016; 19: 115-117. 